\title{
Journal of Drug Metabolism \& Toxicology
}

\section{Identification of Novel Metabolic Pathways of Sitagliptin (STG) by LC/MS and LC/MS2 after Incubations with Rat Hepatocytes}

Osama IG Khreit ${ }^{1^{*}}$, Helen M Grant ${ }^{2}$, Catherine Henderson ${ }^{2}$, David G Watson ${ }^{3^{*}}$ and Oliver B Sutcliffec ${ }^{4}$

${ }^{1}$ Department of Pharmacology and Toxicology, Omar Almukhtar University, Elbeida City, Libya

${ }^{2}$ Department of Biomedical Engineering, University of Strathclyde, Glasgow, UK

${ }^{3}$ Strathclyde Institute of Pharmacy and Biomedical Sciences (SIPBS), University of Strathclyde, Glasgow, UK

${ }^{4}$ School of Science and the Environment, Manchester Metropolitan University, Manchester, UK

"Corresponding authors: Osama IG Khreit, Department of Pharmacology and Toxicology, Faculty of Veterinary Medicine, Omar Almukhtar University, Elbeida City, Libya, Tel: 218-0-91-218-11806; E-mail: osama.khreit@omu.edu.ly

David G Watson, Strathclyde Institute of Pharmacy and Biomedical Sciences (SIPBS), University of Strathclyde, 161 Cathedral Street, Glasgow, UK, Tel: 44-0-141-548-2651; E-mail: d.g.watson@strath.ac.uk

Received date: Nov 24, 2016; Accepted date: Dec 23, 2016; Published date: Dec 30, 2016

Copyright: ๑ 2016 Khreit OIG, et al. This is an open-access article distributed under the terms of the Creative Commons Attribution License, which permits unrestricted use, distribution, and reproduction in any medium, provided the original author and source are credited.

\begin{abstract}
Sitagliptin (STG), a drug for treating Type li Diabetes Mellitus (T2DM), has been associated with severe joint pain in some patients. In this paper the metabolic profile of the drug has been investigated in order to determine metabolism and formation of reactive compounds which may contribute to this adverse effect. Metabolism of STG was investigated in vitro by incubation with freshly isolated Sprague-Dawley rat hepatocytes, to characterize Phase I and II metabolites, and the reaction mixture analysed on a zwitter ionic hydrophilic interaction (ZIC ${ }^{{ }}$-HILIC) column using LC-MS and LC-MS ${ }^{2}$ utilising electrospray ionization (ESI) in the positive ion mode.
\end{abstract}

STG was metabolised to yield eleven metabolites, but in total only $3.1 \%$ of the parent drug was metabolised over $2 \mathrm{hrs}$ incubation. These metabolites were structurally characterized on the basis of accurate mass analyses and the major metabolic routes for STG determined to be via aromatic oxidation $(0.86 \%)$ and desaturation of $\mathrm{N}-\mathrm{C}$ and $\mathrm{C}-\mathrm{C}$ of the piperazine $(0.44 \%)$.

Novel metabolites of STG detected using these methods included STG N-glucuronide (M6) and a di-ketone metabolite (M4), hydroxylation of both the amine group and aromatic ring followed by formation of glucuronide metabolites (M5, M5'), oxidative desaturation of $\mathrm{NH}_{2}$ and di-hydroxylation of metabolites followed by loss of $\mathrm{HF}$.

Also, observed was an $\mathrm{N}$-sulfate metabolite $(0.07 \%)$ and acetylation followed by glucuronide conjugation was also found in trace amounts $(<0.01 \%)$. $\mathrm{MS}^{2}$ fragment ions provide additional structural confirmation providing a possible structure for most metabolites such as by fragment ion loss of the glucuronide group (176 Da) from metabolite M5 and loss of the phenolic sulfate (80 Da) of N-Sulfate metabolite (M7).

Reduction reaction of piperazine ring probably generates highly electrophilic metabolite of STG, which may be susceptible to produce adverse effects. Furthermore, $\mathrm{N}$-oxidation reaction forming reactive intermediates metabolic to give a hydroxylamine metabolite that may undergo further reactions to yield electrophilic intermediate metabolites.

Keywords: STG; DPP-4: Dipeptidyl peptidase 4; Metabolism; ZICHILIC; Mass spectrometry

\section{Abbreviations:}

STG: Sitagliptin; LC/MS: Liquid Chromatography/Mass Spectrometry; LC-MS2: Liquid Chromatography-Multiple Mass Spectrometry; ESI: Electrospray Ionization; CID: Collision-Induced Dissociation; T2DM: Type 2 Diabetes

\section{Introduction}

Diabetes mellitus is a disorder of metabolism, which is a major public health problem in all parts of the world, and recently the World Health Organization suggested that in all parts of the world almost 3 million deaths annually can be attributed to diabetes. Within 10 years, it is expected that about 333 million people world-wide will suffer from diabetes mellitus; with Type 2 Diabetes Mellitus (T2DM) representing about $90-95 \%$ of cases diagnosed [1]

STG is an antidiabetic agent of the Dipeptidyl Peptidase-Iv Inhibitor (DPP-IV) class [2]. It was approved by the Food and Drug Administration (FDA) in October 2006 and produced as a single therapy to treat T2DM by Merck Pharmaceutical Company under the trade name Januvia. These DPP-IV inhibitors are used as monotherapy, and as add-on therapy in patients with T2DM. The FDA agreed to the sitagliptin/metformin (Janumet ${ }^{\mathrm{TM}}$, Merck \& Co.) combination in April 2007 as an assistive treatment in combination with diet and exercise to improve control of blood sugar in adult patients suffering from T2DM [3-5].

Recently, the FDA warned that some T2DM medicines such as STG caused severe joint pain; these symptoms were relieved after the 
Page 2 of 7

patient discontinued the drugs. At present the mechanism responsible for this adverse effect is not known, either in terms of the chemical species involved or the physiological process affected. Understanding the metabolic pathways and formation of reactive metabolites will aid understanding of in the vivo metabolism, likely pharmacokinetics and adverse effects of this class of medicine [6]. The chemical structure of STG is shown on Figure 1.

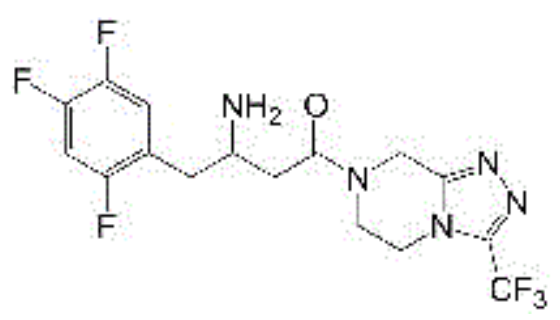

Figure 1: Chemical structure of SGT.

In the literature, there are Gas Chromatography-Mass Spectrometry (GC-MS) methods for the determination of STG in biological samples. However, these chromatographic studies require a complicated process to enable identification of trace amounts of STG metabolites using drying and then derivatisation by $\mathrm{N}$ methyltrimethylsilyltrifluoroacetamide prior to GC-MS analysis and conversion into its N-TMS amine derivative, and these processes might affect the stability of some metabolites which are formed in trace amounts or indeed they may simply not be volatile enough for GC-MS analysis [7]. On the other hand, Vincent et al. recently reported the phase I and II metabolism of $\left[{ }^{14} \mathrm{C}\right]$ STG and six metabolites at trace levels in human plasma, urine and faeces using LC-MS $^{2}$ and radiometric detection using the TurboIonSpray interface operated in the positive ion mode. Six phase I and II metabolites of STG were observed in both human urine and faeces and these were shown to be products of hydroxylated, N-sulfated STG and two metabolites formed by oxidative desaturation of the piperazine ring followed by cyclization. It was observed that, in all human subjects, the glucuronides were further transformed and were not detected in faeces samples [8].

Similar observations were shown in rats and dogs, where, as in humans, approximately $10-16 \%$ of the radiolabeled oral dose was recovered in the rat and dog excreta as phase I and II metabolites. All the metabolites observed in human plasma, urine, and faeces were also observed in rat and dog plasma, urine, bile, and faeces, as well as in incubations in vitro with rat, dog, and human liver microsomes. However, metabolism was minimal in all species in vitro and in vivo. Results from in vitro experiments with recombinant P450s and monoclonal anti-P450 antibodies showed that the oxidative metabolite of STG in human liver microsomes is catalysed primarily by CYP3A4 with some minor contribution from CYP2C8 [9]. The authors did not detect any other products arising from Phase II metabolism except the glucuronide and sulfate metabolites.

As knowledge of the ADME processes for reactive metabolites is a prerequisite for their toxicological risk assessment it is prudent to elucidate both the phase I and II metabolic pathways for STG especially in the long term administration for this kind of medication. This paper seeks to address this deficiency by presenting the elucidation and profile of both phase I and phase II metabolic events in rat hepatocyte preparations using LC-MS and LC-MS ${ }^{2}$ methods.

\section{Experimental}

\section{Chemical and reagents}

All reagents were of commercial quality (obtained from SigmaAldrich, Gillingham, UK, Alfa-Aesar, Heysham, UK or Fisher Scientific, Loughborough, UK) and used without further purification. Sitagliptin phosphate (STG) was obtained from Kemprotec Limited, UK. HPLC grade acetonitrile was obtained from Fisher Scientific, UK, and formic acid (98\%) was obtained from BDH (DOREST, UK).

The LC separation was performed using a Exactive Orbitrap (Thermo Fisher Scientific, Hemel Hempstead, UK), which was operated in the positive mode, A ZIC-HILIC Column 5 um $150 \mathrm{~mm} \times$ $4.6 \mathrm{~mm}$ (HiChrom, Reading, UK), and acquisition was performed using XCalibur software. Fragmentation experiments were carried out using LTQ Orbitrap (Thermo Fisher Scientific, Hemel Hempstead, $\mathrm{UK})$.

\section{Data processing}

LC/MS raw files were processed by Thermo Sieve software which can provide variances in the plots, which were highlighted, and the exact masses of ions yielding the difference could be detected. The parameters used in this software were: time range 4-30 min, mass range 75-800 amu, frame width $0.02 \mathrm{amu}$ and Rt width $2.5 \mathrm{~min}$ [10]. It can be shown clearly that this software detected a difference in the intensity peaks of the metabolite ions between STG incubated with hepatocytes for $120 \mathrm{~min}$ and STG, incubated with buffer alone, and hepatocytes incubated in the absence of STG after $120 \mathrm{~min}$.

\section{Biotransformation of STG in Sprague-Dawley rat hepatocytes}

All animal procedures adhered to UK Home Office Guidelines and were carried out under Licence PPL/60/3685. The hepatocytes were prepared from male Sprague-Dawley rats (body weight range: 200-250 $\mathrm{g}$, bred in the University of Strathclyde) by collagenase perfusion of the livers using a modification of the method by Moldeus et al. [11,12]. The cell viability was confirmed using a Trypan blue exclusion test, and was $>80 \%$. STG $(100 \mu \mathrm{M}$, a standard concentration used to screen metabolic pathways) was incubated in $50 \mathrm{~mL}$ round-bottomed flasks with hepatocytes $\left(2 \times 10^{6}\right.$ viable cells $\left.\mathrm{mL}^{-1}\right)$ in Krebs-Henseleit $(\mathrm{K}-\mathrm{H})$ buffer containing $12.5 \mathrm{mM}$ HEPES $(\mathrm{pH}$ 7.4). The samples were incubated with constant rotation at $37^{\circ} \mathrm{C}$ under an atmosphere of oxygen: carbon dioxide (95:5) for $120 \mathrm{~min}$. Control incubations of (i) rat hepatocytes (in the absence of STG) and (ii) solutions of (STG) dissolved in K-H buffer (pH7.4) was also carried out. All incubations were carried out in triplicate, using three independent animals.

\section{Sample preparation}

Aliquots $(0.5 \mathrm{~mL})$ were removed at $0 \mathrm{~min}, 30 \mathrm{~min}$, and $120 \mathrm{~min}$ from the incubations, and quenched by addition of acetonitrile $(1 \mathrm{~mL})$. The samples were centrifuged $\left(10^{4} \mathrm{rpm}, 15 \mathrm{~min}\right)$ and any remaining protein removed by further addition of acetonitrile $(0.2 \mathrm{~mL})$ and subsequent filtration through a Biotage Isolute ${ }^{\circ} \mathrm{PPT}+$ protein precipitation plate (Biotage Limited, Sweden). These crash plates are attached to a vacuum manifold, and each sample mixed with $0.5 \mathrm{~mL}$ of acetonitrile to allow the protein to precipitate, then the filtrate was collected and analysed by either LC-MS or LC-MS². 
Citation: Khreit OIG, Grant HM, Henderson C, Watson DG, Sutcliffe OB (2016) Identification of Novel Metabolic Pathways of Sitagliptin (STG) by LC/MS and LC/MS2 after Incubations with Rat Hepatocytes. J Drug Metab Toxicol 7: 220. doi:10.4172/2157-7609.1000220

Page 3 of 7

The efficiency of recovery from hepatocyte matrix was determined to be $102.6 \% \pm 20.7 \%$ (for STG, $n=3, \pm S D$ )

\section{Liquid chromatography-mass spectrometry (LC-MS)}

Liquid chromatography-mass spectrometry (LC-MS) data were acquired using an Orbitrap Exactive instrument (Thermo-Fisher Corporation, Hemel Hempstead, UK). Sample analysis was carried out with positive ion ESI detection. The mass scanning range was 75-1200 $\mathrm{m} / \mathrm{z}$, while the capillary temperature was $250^{\circ} \mathrm{C}$, spray voltage was +4.5 $\mathrm{kV}$ and the sheath and auxiliary gas (nitrogen) flow rates were 45 and 15 , respectively (units not specified by the manufacturer). The LC-MS system (controlled by Xcalibur Ver. 2.0, Thermo-Fisher Corporation, Hemel Hempstead, UK) was run in binary gradient mode with an injection volume of $10 \mu \mathrm{L}$. Solvent A was aqueous formic acid $(0.1 \%$ $\mathrm{v} / \mathrm{v})$ and solvent $\mathrm{B}$ was formic acid $(0.1 \% \mathrm{v} / \mathrm{v})$ in acetonitrile; the flow rate was $0.3 \mathrm{~mL} \mathrm{~min}^{-1}$. A ZIC ${ }^{\circ}$-HILIC $(150 \mathrm{~mm} \times 4.6 \mathrm{~mm}$ i.d., particle size: $5 \mu \mathrm{m}$ ) column fitted with a ZIC ${ }^{\circ}$-HILIC guard column (HiChrom Limited, Reading, UK) was used for all analyses. The gradient programme was as follows: $20 \% \mathrm{~A}(0 \mathrm{~min})$ to $50 \% \mathrm{~A}$ (at $12 \mathrm{~min}$ ), to $50 \% \mathrm{~A}$ (at $26 \mathrm{~min}$ ), to $80 \% \mathrm{~A}$ (at $28 \mathrm{~min}$ ), to $80 \% \mathrm{~A}$ (at $36 \mathrm{~min}$ ), to $20 \%$ A (at $37 \mathrm{~min}$ ) and finally re-equilibration with $20 \%$ A to $45 \mathrm{~min}$. LC$\mathrm{MS}^{2}$ spectra were obtained by using an LTQ Orbitrap (Thermo-Fisher Corporation, Hemel Hempstead, UK) with the same source, control software and chromatographic conditions specified above. The selected precursor ions were fragmented with a CID voltage of $40 \mathrm{eV}$. Data processing was carried out using SIEVE Ver. 1.2.1 (ThermoFisher,
Hemel Hempstead UK), ToxID Ver. 1.2.1 (ThermoFisher, Hemel Hempstead UK) and MetWorks Ver. 1.3 (ThermoFisher, Hemel Hempstead UK) software.

\section{Results and Discussion}

\section{Confirmation of Sprague-Dawley rat hepatocyte viability}

The viability of the freshly isolated hepatocytes was $79.5 \pm 4.6 \%$, and exposure to STG had no significant effect on viability compared with cells incubated in K-H buffer alone for up to $2 \mathrm{hrs}$.

\section{Biotransformation of STG in Sprague-Dawley rat hepatocytes}

Using the high resolution mass spectrometer, 11 potential STG metabolites were detected by using SIEVE software to compare the control incubation with the hepatocyte incubation containing STG. Only $3.1 \% \pm 0.12 \%$ of the parent STG was metabolised by the end of the two hr incubation. Five of the eleven metabolites have been previously identified [8], along with products arising from sulfation, glucuronidation, acetylation, hydroxylation and other pathways [9].

Thus it was found that rat hepatocytes catalysed both phase I and phase II metabolism of STG and overall eleven metabolites were putatively identified (Table 1).

\begin{tabular}{|c|c|c|c|c|c|}
\hline Metabolite & Rt (min) & $\mathrm{m} / \mathrm{z}$ & Formula (ppm) & MS2 Base Peak & Other lons \\
\hline STG & 9.6 & 408.13 & $\mathrm{C}_{16} \mathrm{H}_{16} \mathrm{~F}_{6} \mathrm{~N}_{5} \mathrm{O}(0.224)$ & 235 & 193(26),391(9),174(4) \\
\hline M1 & 8.25 & 424.12 & $\mathrm{C}_{16} \mathrm{H}_{16} \mathrm{~F}_{6} \mathrm{~N}_{5} \mathrm{O}_{2}(-0.001)$ & 391 & $193(12), 235(2)$ \\
\hline M1' & $9.7,10.1$ & 424.12 & $\mathrm{C}_{16} \mathrm{H}_{16} \mathrm{~F}_{6} \mathrm{~N}_{5} \mathrm{O}_{2}(-0.001)$ & 406 & 235(6),191(5),193(3) \\
\hline M2 & 9.72 & 406.11 & $\mathrm{C}_{16} \mathrm{H}_{14} \mathrm{~F}_{6} \mathrm{~N}_{5} \mathrm{O}(-0.3134)$ & 191 & $174(6), 233(4)$ \\
\hline M2' & 5.67 & 406.11 & $\mathrm{C}_{16} \mathrm{H}_{14} \mathrm{~F}_{6} \mathrm{~N}_{5} \mathrm{O}(0.206)$ & 191 & $174(6)$ \\
\hline M3 & 5.67 & 422.10 & $\mathrm{C}_{16} \mathrm{H}_{14} \mathrm{~F}_{6} \mathrm{~N}_{5} \mathrm{O}_{2}(0.781)$ & 193 & $404(96), 389(63), 285(49), 191(44), 207(10)$ \\
\hline M4 & 5.6 & 407.09 & $\mathrm{C}_{16} \mathrm{H}_{13} \mathrm{~F}_{6} \mathrm{~N}_{4} \mathrm{O}_{2}(0.026)$ & 193 & $191(16), 192(11), 389(1)$ \\
\hline M5 & 6.4 & 600.15 & $\mathrm{C}_{22} \mathrm{H}_{24} \mathrm{~F}_{6} \mathrm{~N}_{5} \mathrm{O}_{8}(-0.230)$ & 424(-gluc) & $\begin{array}{l}582(55), 391(26), 191(10), 193(10), \\
408(8)\end{array}$ \\
\hline M5'- & $9.95,11.1$ & 600.15 & $\mathrm{C}_{22} \mathrm{H}_{24} \mathrm{~F}_{6} \mathrm{~N}_{5} \mathrm{O}_{8}(-0.430)$ & 406 & $582(5)(-\mathrm{H} 2 \mathrm{O}), 424(3)$ \\
\hline M6 & 9.34 & 584.16 & $\mathrm{C}_{22} \mathrm{H}_{24} \mathrm{~F}_{6} \mathrm{~N}_{5} \mathrm{O}_{7}(0.353)$ & $\begin{array}{l}530 \\
(-3 \mathrm{H} 2 \mathrm{O})\end{array}$ & 486(53),408(7,-gluc) \\
\hline M7 & 5.12 & 488.08 & $\mathrm{C}_{16} \mathrm{H}_{16} \mathrm{~F}_{6} \mathrm{~N}_{5} \mathrm{O}_{4} \mathrm{~S}(-0.11)$ & 408(-SO3) & $470(3)(-\mathrm{H} 2 \mathrm{O})$ \\
\hline M8 & 6.26 & 452.12 & $\mathrm{C}_{17} \mathrm{H}_{16} \mathrm{~F}_{6} \mathrm{~N}_{5} \mathrm{O}_{3}(-0.025)$ & ND & \\
\hline M9 & 6.3 & 628.15 & $\mathrm{C}_{23} \mathrm{H}_{24} \mathrm{~F}_{6} \mathrm{~N}_{5} \mathrm{O}_{9}(-0.383)$ & 516 & $\begin{array}{l}\text { 408(30),452(28,-gluc),404(20), } \\
610(19,-\mathrm{H} 2 \mathrm{O})\end{array}$ \\
\hline M10 & 5.8 & 440.12 & $\mathrm{C}_{16} \mathrm{H}_{16} \mathrm{~F}_{6} \mathrm{~N}_{5} \mathrm{O}_{3}(0.147)$ & $422(-\mathrm{H} 2 \mathrm{O})$ & \\
\hline M11 & 10.7 & 422.12 & $\mathrm{C}_{16} \mathrm{H}_{17} \mathrm{~F}_{5} \mathrm{~N}_{5} \mathrm{O}_{3}(-0.187)$ & $404(\mathrm{H} 2 \mathrm{O})$ & \\
\hline
\end{tabular}

Table 1: Phase I and phase II metabolism of STG. 
The elemental composition and retention times of the metabolites are listed in Table 1. The mass error (when compared to their theoretical masses) of all the detected metabolites were within $\pm 0.5-1$ ppm of their assigned elemental composition (Table 1), however, the most abundant metabolites after 30 mins incubation were proposed to be aromatic hydroxylation metabolites $\left(\mathrm{Ml}, \mathrm{t}_{\mathrm{R}}=9.7 \mathrm{~min} / 10.1 \mathrm{~min} ; \mathrm{m} /\right.$ $\mathrm{z}=424.12$ ), and desaturated metabolites formed through desaturation of $\mathrm{N}-\mathrm{C}$ and $\mathrm{C}-\mathrm{C}$ of the piperazine ring ( $\mathrm{M} 2$ and $\mathrm{M} 2$ ' respectively, $t_{R}=9.7 \mathrm{~min}, 5.7 \mathrm{~min} ; \mathrm{m} / \mathrm{z}=406.11$ ). M2 was formed from STG by conversion of a tertiary amide to a quaternary amide via $\mathrm{C}-\mathrm{N}$ desaturation. The mass spectral fragmentation pattern (Figure 2) shown included fragment ion $(\mathrm{m} / \mathrm{z} 191)$, and Liu showed similar findings and obtained the same fragmentation pattern. Thus M2 was formed through desaturation of C-N and M2' through desaturation C$\mathrm{C}$ of piperazine ring [13].
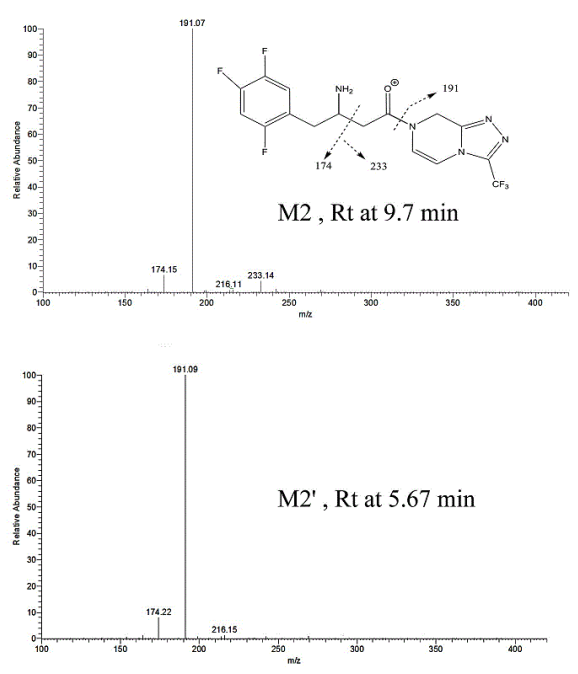

Figure 2: The mass spectral fragmentation pattern.

The levels of most of these metabolites increased further between 30 and $120 \mathrm{~min}$ of incubation with a corresponding slight decrease in the level of unmetabolised drug ( $\left.S T G, t_{R}=9.6 \mathrm{~min}\right)$.

In contrast, the level of $\left(M 8, t_{R}=6.3 \mathrm{~min} ; \mathrm{m} / \mathrm{z}=452.12\right)$ was similar between $30 \mathrm{~min}$ and $120 \mathrm{~min}$ and there was an increase in the levels of its corresponding glucuronide $\left(\mathrm{M} 9, \mathrm{t}_{\mathrm{R}}=6.5 \mathrm{~min} ; \mathrm{m} / \mathrm{z}=628.15\right)$. The sulfate metabolite $\left(M 7, t_{R}=5.12 \mathrm{~min} ; \mathrm{m} / \mathrm{z}=488.08\right)$ could also be clearly observed after $120 \mathrm{~min}$ of incubation and it is considered a stable nontoxic conjugate. In some cases chromatographic information can be used to differentiate isomeric possibilities. Thus metabolite (M1) is proposed to result from hydroxylation on the amine group at the nucleophilic nitrogen forming a hydroxylamine rather than hydroxylation of the aromatic moiety of STG (M1'), M1 and M1' elute at a different retention time $\left(t_{R}=8.25 \mathrm{~min}, t_{R}=9.7 ; 10.1 \mathrm{~min}\right.$ respectively) on the ZIC'-HILIC column.

The biotransformation of (STG) observed following its incubation with Sprague-Dawley rat hepatocytes is summarised. The dynamic range of the instrument was sufficient to show both major and minor metabolites. Metabolite M1 and M1', eluted at 8.25 and $9.7 \mathrm{~min}$, and had a protonated molecular ion $[\mathrm{M}+\mathrm{H}]^{+}$at $\mathrm{m} / \mathrm{z} 424.12$ which indicates possible hydroxylation at $\mathrm{NH}_{2}$ (M1) as the largest fragment ion was observed at $\mathrm{m} / \mathrm{z} 391$, which can be attributed to the loss of $\left(\mathrm{NH}_{3}-\mathrm{OH}\right)$ or loss of $18 \mathrm{D}$ at the aromatic position metabolite (M1') to produce fragment ion $\mathrm{m} / \mathrm{z} 406$.

Moreover, metabolite M2 and M2', showed the same protonated molecular ion $[\mathrm{M}+\mathrm{H}]^{+}$at $\mathrm{m} / \mathrm{z}$ 406.1; the molecular ions were $2 \mathrm{Da}$ less than that of STG parent ion, indicating loss of 2 Hydrogen atoms. Due to the lack of reference standards in this study the results could not define the exact desaturation positions of M2 and M2'. However, the advantage of the high resolution of mass spectrometry in assignment of the largest fragment at $\mathrm{m} / \mathrm{z} 191$ showed the two possible structures of these metabolites as desaturated in $\mathrm{C}-\mathrm{C}$ and $\mathrm{C}-\mathrm{N}$ of the piperazine ring (Figure 2). In addition, the results of this study indicate that STG is metabolised to an electrophilic form (M2) which is an ionized metabolite, and it may be implicated in adverse effects of STG, as it is an electrophilic entity capable of irreversible reaction with tissue nucleophiles [14]

When the primary amine underwent $\mathrm{N}$-oxidation it formed a hydroxylamine. This provides a pathway for metabolic deamination. Thus STG is transformed to a hydroxylamine that then underwent dehydration to an imine. This is subjected to further metabolic oxidation to give an oxime because the imine had hydrogen attached to the nitrogen (M3, $\mathrm{m} / \mathrm{z} 422.10)$. The carbon of the oxime is electrophilic and water attacks it to form a carbinolhydroxylamine that is then hydrolyzed to form a ketone and hydroxylamine. Thus M4 has a protonated molecular ion $[\mathrm{M}+\mathrm{H}]^{+}$at $\mathrm{m} / \mathrm{z} 407.09$ and was identified as a deamination metabolite of STG (Scheme 1) [15].

Several enzyme systems such as CYP dependent FMOs can catalyze $\mathrm{N}$-Oxidation reactions $[16,17]$ and this pathway has the potential to lead to metabolites that can damage cells via multiple pathways. Metabolites, such as the N-hydroxyl metabolites, can redox cycle to produce reactive oxygen species and other free radicals, which can damage membranes and lead to oxidative stress $[18,19]$.

The primary amine group in STG reacted to form $\mathrm{N}$ - glucuronide metabolites $(\mathrm{M} 6, \mathrm{tR}=9.3 \mathrm{~min} ; \mathrm{m} / \mathrm{z}=584.15)$. The fragmentation pattern showed a mass shift of $176 \mathrm{Da}$ compared with the parent STG, because the amine nucleophilic functional groups react at the acetal carbon of the sugar, inverting the configuration at that site to give an $\mathrm{N}$ glucuronide conjugate. This reaction is catalyzed by UDP-glucuronyl transferase. The hydroxylamine group in $\mathrm{M} 1$ also can react to form $\mathrm{O}$ glucuronide metabolites ( $\mathrm{M} 5, \mathrm{M} 5, \mathrm{t}_{\mathrm{R}}=6.4,10$ and $11 \mathrm{~min} ; \mathrm{m} /$ $\mathrm{z}=600.11$ ). Glucuronide formation is an important step in the elimination of many important exogenous substances from the body; the major glucuronide metabolites observed in the current work were M5 and M6. There was also a glucuronide as a minor metabolite, an $\mathrm{N}$ carbamoyl glucuronidation $\left(\mathrm{M} 9, \mathrm{t}_{\mathrm{R}}=6.5 \mathrm{~min} ; \mathrm{m} / \mathrm{z}=628.15\right)$.

Karabulut et al. reported that the number of apoptotic cells was the same in the pancreas of healthy and type-2 diabetic rats after STG administration. Serum glutathione levels did not change in either group. Glutathione conjugated metabolites were not detected in our study [20], however, the $\mathrm{N}$-oxide metabolite needs further study to fully characterise its pharmacological/toxicological effects.

Defluorination of STG occurred forming (M11, $t_{R}=10.4 \mathrm{~min} ; \mathrm{m} /$ $\mathrm{Z}=422.12$ ) in trace amounts after two hrs of incubation, presumably after nitrogen and aromatic hydroxylation $\left(\mathrm{M} 10, \mathrm{t}_{\mathrm{R}}=9.8 \mathrm{~min} ; \mathrm{m} /\right.$ $\mathrm{z}=440.12$ ). 


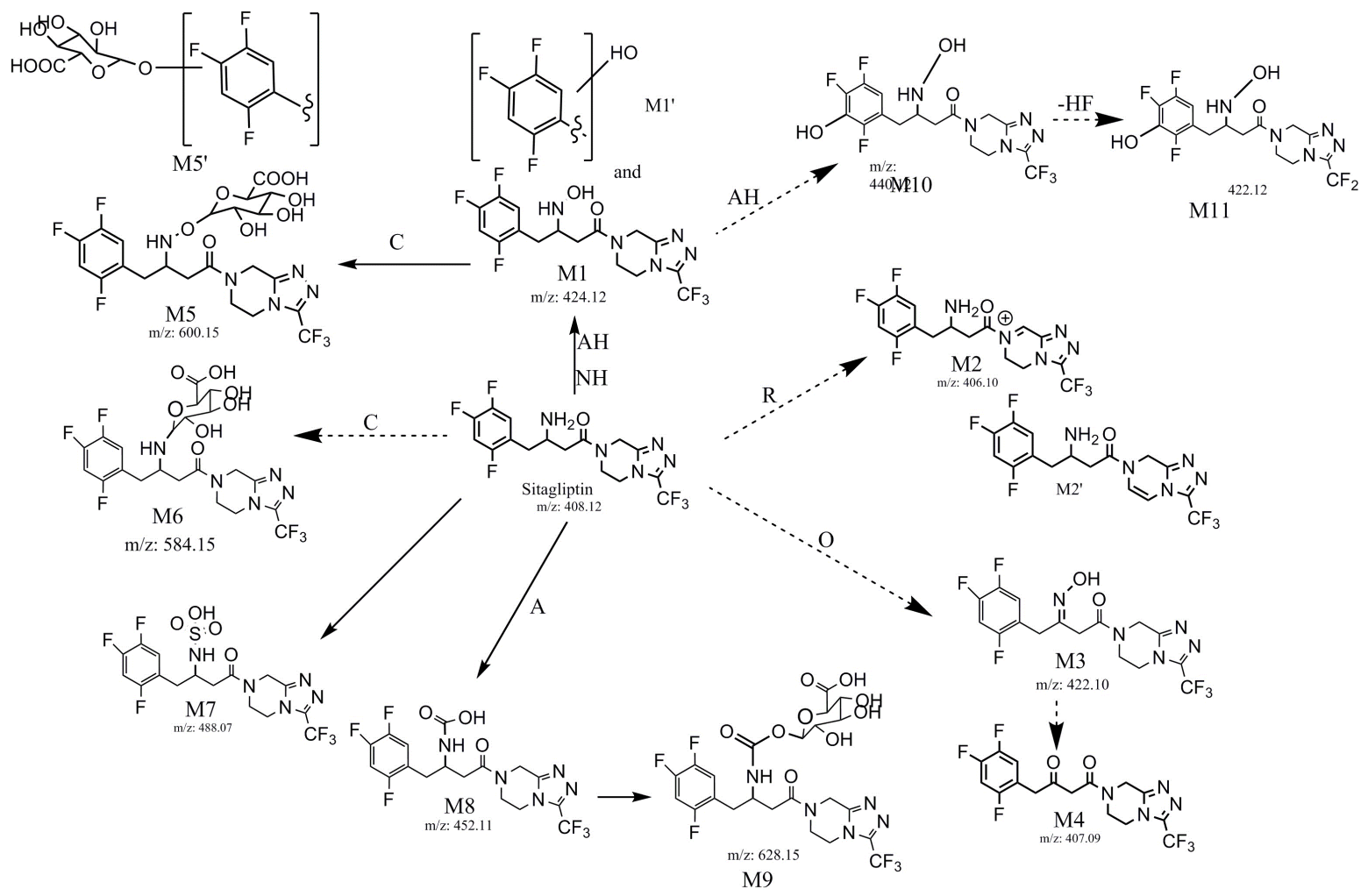

Scheme 1: Deamination metabolite of STG.

\section{Metabolic profiling of Sitagliptin (STG)}

Drugs are eliminated from the body by excretion and by transformation to metabolites. These processes occur simultaneously, but which of these processes predominate varies from one drug to another. As the amount of drug in a system depends on both its rates of absorption and elimination and the amount of a specific metabolite present also depends on its rate of formation and elimination. The metabolic profiles of STG ( $\mathrm{pKa}=8.78$; $\log \mathrm{P}=1.26$ ) were determined using three separate incubations of the drugs with isolated rat hepatocytes (Figure 3).

The relative levels of the detected metabolites of STG indicate that eleven metabolites were identified in the freshly isolated cells. The product-ion spectrum of protonation recorded at $40 \mathrm{eV}$ collision energy is shown in Figure 3 and only aromatic hydroxylation STG (M1', $\mathrm{m} / \mathrm{z}=424.12$ ) was formed in large amounts. In addition, the formation of metabolite $(\mathrm{M} 5, \mathrm{~m} / \mathrm{z}=600.15)$ increased after two hrs incubation period by approximately $100 \%$. In the case of (STG) the histogram indicates that the major metabolites of STG were: (M1', m/ $\mathrm{z}=424.11),(\mathrm{M} 2, \mathrm{~m} / \mathrm{z}=406.10),(\mathrm{M} 4, \mathrm{~m} / \mathrm{z}=407.09)$ and $(\mathrm{M} 5, \mathrm{~m} /$ $\mathrm{z}=600.15$ ) (Figure 3 ). The amount of (M3) and (M6) increased slowly throughout the two hr incubation period. The majority of Phase II metabolites identified in (STG) were found at low levels at $30 \mathrm{~min}$ and increased slightly by $120 \mathrm{~min}$. The $\mathrm{N}$-sulfate metabolite (M7, m/ $\mathrm{z}=488.08$ ) increased slowly though the level was low at 30 as well as $120 \mathrm{~min}$, moreover (M8, $\mathrm{m} / \mathrm{z}=452.12),(\mathrm{M} 9 \mathrm{~m} / \mathrm{z}=628.15)$ and $(\mathrm{M} 11 \mathrm{~m} /$ $\mathrm{z}=422.12$ ) were identified at only trace amounts, however, the sensitivity and high resolution of the instrument was sufficient to show these minor metabolites.

In a similar case in a healthy male, Herman showed that almost all STG was excreted unchanged in urine and faeces after oral administration. It was eliminated $87 \%$ in urine and $13 \%$ in the faeces. Renal clearance involving active tubular secretion was the main elimination pathway [21]. In our study, as can be seen from Table 1, the results obtained from the preliminary analysis of rat hepatocyte incubations showed both phase I and phase II metabolism of STG. However, the metabolism of STG in liver cells was also minimal. $\mathrm{M}+\mathrm{H}$ was the major metabolite formed. A considerable amount of literature has been published on STG metabolism. All the metabolites reported in previous studies have been produced in our study when hepatocytes were incubated with STG, except for the glutathione conjugate reported by Beconi [9] which was absent in our study.

The most obvious finding to emerge from the analysis is that total metabolism of STG was only about 3\%. It can thus be suggested that the adverse effects that were reported recently may be caused by the parent drug. This view is supported by Swallow who reported that high metabolic stability and bioavailability of STG of $87 \%$ were demonstrated in vivo in healthy volunteers and the author showed that renal clearance of unchanged STG accounted for approximately $70 \%$ of the total plasma clearance of STG. Therefore, further studies of the adverse effects of STG which take these variables into account, will need to be undertaken $[22,23]$. 
Citation: Khreit OIG, Grant HM, Henderson C, Watson DG, Sutcliffe OB (2016) Identification of Novel Metabolic Pathways of Sitagliptin (STG) by LC/MS and LC/MS2 after Incubations with Rat Hepatocytes. J Drug Metab Toxicol 7: 220. doi:10.4172/2157-7609.1000220

Page 6 of 7

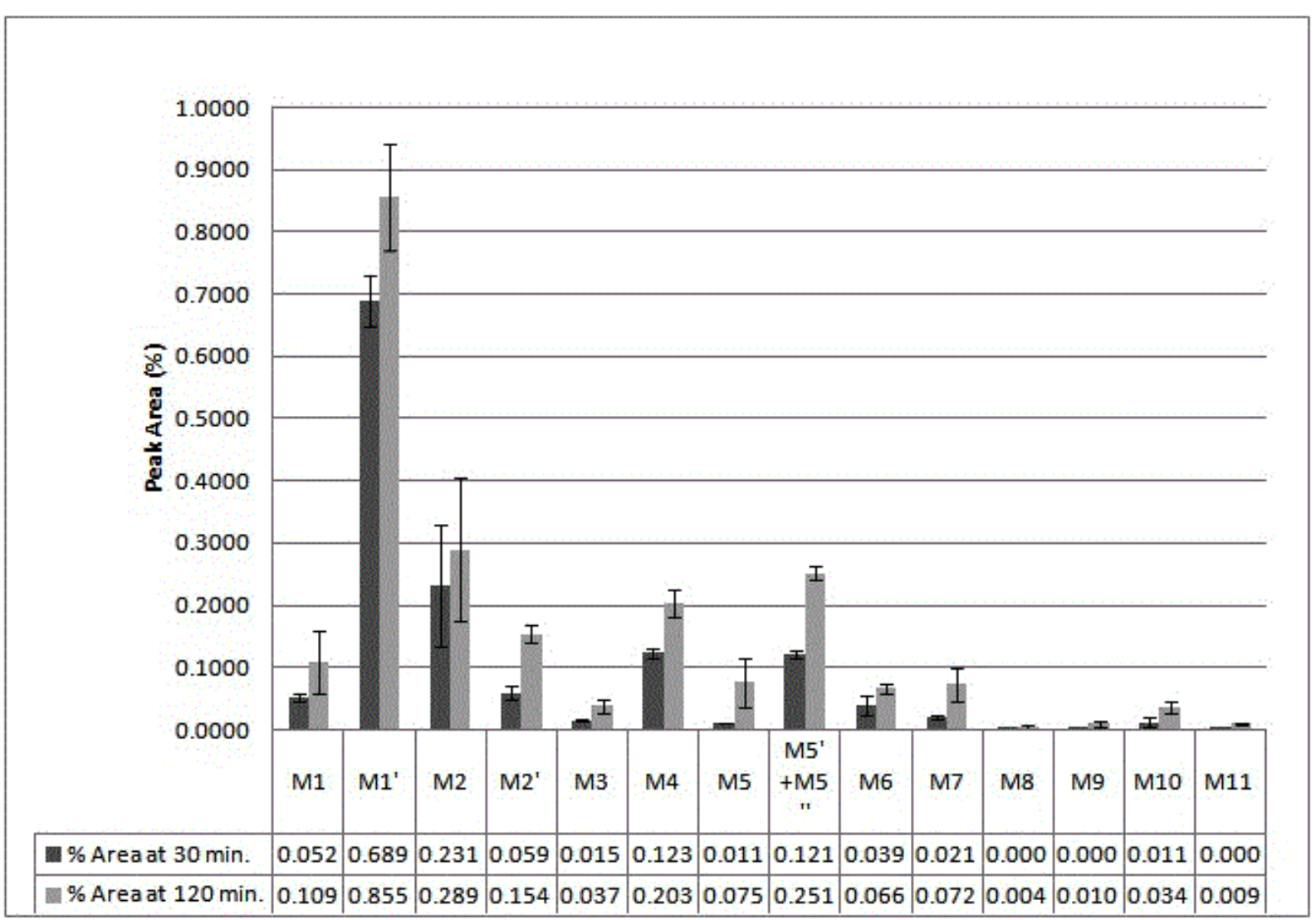

Figure 3: The metabolic profiles of STG ( $\mathrm{pKa}=8.78$; $\log \mathrm{P}=1.26$ ) were determined using three separate incubations of the drugs with isolated rat hepatocytes.

\section{Conclusion}

The data presented both confirm and expand upon previously reported observations through the identification of all Phase II metabolites, however, no evidence of the formation of ring cyclization after oxidative desaturation of the piperazine were detected in the current study. STG shows some novel metabolite pathways such as a deaminated metabolite in which the amino group is replaced by oxygen. The key pharmacokinetic parameters for STG reported herein may be of significance to medical pharmacologists and can be used to predict both the in vivo metabolism and the likely pharmacokinetic effects of STG within this class of anti-diabetic substances [24].

The present study confirms previous findings of STG metabolism. This study did not confirm formation of a glutathione conjugate metabolite, and for those new metabolites such as diketone metabolite of STG, N-hydroxylation and oxime metabolites, further studies are needed to investigate the pharmacological evaluation.

\section{Acknowledgements}

The authors gratefully acknowledge Dr Gavin J. Blackburn (University of Strathclyde, SIPBS) and Dr Mohammed AL bratty (Saudi Arabia, Guzan University) for assistance with data analysis and laboratory support for this work through University of Strathclyde.

\section{References}

1. Wua JYC, Shia X, Gua W (2009) Dipeptidyl peptidase IV (DPP IV): a novel emerging target for the treatment of type 2 diabetes. J Nanjing Med Univ 23: 228-235.

2. Lankas GR (2005) Dipeptidyl peptidase IV inhibition for the treatment of type 2 diabetes: potential importance of selectivity over dipeptidyl peptidases 8 and 9. Diabetes 54: 2988-2994.

3. Charbonnel B (2006) Efficacy and safety of the dipeptidyl peptidase-4 inhibitor sitagliptin added to ongoing metformin therapy in patients with type 2 diabetes inadequately controlled with metformin alone. Diabetes Care 29: 2638-2643.

4. Idris I, Donnelly R (2007) Dipeptidyl peptidase-IV inhibitors: a major new class of oral antidiabetic drug. Diabetes Obes Metab 9: 153-165.

5. Choy M, Lam S (2007) Sitagliptin: a novel drug for the treatment of type 2 diabetes. Cardiol Rev 15: 264-271.

6. http://www.fda.gov/Drugs/DrugSafety/ucm459579

7. Uçaktürk E (2013) Development of a gas chromatography-mass spectrometry method for the analysis of sitagliptin in human urine. J Pharm Biomed Anal 74: 71-76.

8. Vincent SH (2007) Metabolism and excretion of the dipeptidyl peptidase 4 inhibitor [14C] sitagliptin in humans. Drug Metab Dispos 35: 533-538.

9. Beconi MG (2007) Disposition of the dipeptidyl peptidase 4 inhibitor sitagliptin in rats and dogs. Drug Metab Dispos 35: 525-532.

10. Bratty MA (2012) Metabolomic profiling reveals that Drosophila melanogaster larvae with the y mutation have altered lysine metabolism. FEBS Open Bio 2: 217-221. 
Citation: Khreit OIG, Grant HM, Henderson C, Watson DG, Sutcliffe OB (2016) Identification of Novel Metabolic Pathways of Sitagliptin (STG) by LC/MS and LC/MS2 after Incubations with Rat Hepatocytes. J Drug Metab Toxicol 7: 220. doi:10.4172/2157-7609.1000220

Page 7 of 7

11. Moldeus P, Hogberg J, Orrenius S (1978) Isolation and use of liver cells. Methods Enzymol 52: 60-71.

12. Khreit OIG (2013) Elucidation of the Phase I and Phase II metabolic pathways of $( \pm)-4^{\prime}$-methylmethcathinone $(4-\mathrm{MMC})$ and $( \pm)-4^{\prime}-$ (trifluoromethyl)methcathinone (4-TFMMC) in rat liver hepatocytes using LC-MS and LC-MS2. J Pharm Biomed Anal 72: 177-185.

13. Liu DQ (2007) Characterization of Two Cyclic Metabolites of Sitagliptin. Drug Metab Dispos 35: 521-524.

14. Macherey AC, Dansette PM (2008) Chapter 33-Biotransformations Leading to Toxic Metabolites: Chemical Aspect A2-Wermuth, Camille Georges, in The Practice of Medicinal Chemistry (Third Edition) Academic Press: New York, pp: 674-696.

15. Rice JE (2014) Chapter 7-Drug Metabolism. In: Rice JE (ed.) Organic Chemistry Concepts and Applications for Medicinal Chemistry. Academic Press: Boston, pp: 145-201.

16. Yanni SB (2010) In vitro hepatic metabolism explains higher clearance of voriconazole in children versus adults: role of CYP2C19 and flavincontaining monooxygenase 3. Drug Metab Dispos 38: 25-31.

17. Patrick GL (2009) An introduction to medicinal chemistry. Oxford University Press.

18. Valentovic MA (1997) Characterization of methemoglobin formation induced by 3,5-dichloroaniline, 4-amino-2,6-dichlorophenol and 3,5dichlorophenylhydroxylamine. Toxicology 118: 23-36.
19. Racine C (2014) 3,4,5-Trichloroaniline Nephrotoxicity in vitro: Potential Role of Free Radicals and Renal Biotransformation. Int J Mol Sci 15: 20900-20912.

20. Karabulut S, Coskun ZM, Bolkent S (2015) Immunohistochemical, apoptotic and biochemical changes by dipeptidyl peptidase- 4 inhibitorsitagliptin in type-2 diabetic rats. Pharmacol Rep.

21. Herman GA (2005) Pharmacokinetics and pharmacodynamics of sitagliptin, an inhibitor of dipeptidyl peptidase IV, in healthy subjects: results from two randomized, double-blind, placebo-controlled studies with single oral doses. Clin Pharmacol Ther 78: 675-688.

22. Swallow S (2015) Chapter Two-Fluorine in Medicinal Chemistry. In: Lawton G and Witty DR (eds.) Progress in Medicinal Chemistry, Elsevier, pp: 65-133.

23. Bergman A (2007) Absolute bioavailability of sitagliptin, an oral dipeptidyl peptidase- 4 inhibitor, in healthy volunteers. Biopharmaceut Drug Dispos 28: 315-322.

24. Benigni R, Bossa C (2011) Mechanisms of Chemical Carcinogenicity and Mutagenicity: A Review with Implications for Predictive Toxicology. Chem Rev 111: 2507-2536. 